\title{
THE COLLECTED WORKS OF MILLER
}

The Collected Works of George Abram Miller. Vol. 2. By H. R. Brahana, R. D. Carmichael and A. B. Coble, The Committee on Publication, University of Illinois Press, 1938. $11+537 \mathrm{pp}$.

This volume reproduces one hundred seven papers published by Miller during the years from 1900 through 1907. It also contains two papers written by him especially for this volume. One of these (No. 63) is entitled "Note on the History of Group Theory during the Period Covered by this Volume"; the other (No. 171) is entitled "Primary Facts on the History of Mathematics." The second of these was included at Miller's request. In an appendix there are listed the titles of forty other papers written by him during the period; some of these are covered by papers included and the others are concerned with history, elementary illustrations, applications, or expositions. A subject index and a name index prepared by Miller are also included.

The paper entitled "Note on the History of Group Theory during the Period Covered by this Volume," like the three historical notes written for Volume I, gives a good survey of those developments which are closely related to Miller's work. In 1898 Miller published his first report on recent progress in the theory of groups of a finite order. Second and third reports on the same subject were published by him in 1902 and 1907 respectively. The second report (No. 89 in this volume) is devoted chiefly to recent advances in abstract groups, holomorphisms, substitution groups, and group characteristics, and the third (No. 153) is devoted to elementary applications, abstract groups, groups of isomorphisms, and substitution groups. These reports along with the historical papers just mentioned trace in a satisfactory way the development of the theory of groups up to 1908 and afford a splendid basis for the appreciation of the work done in this field up to this time by Professor Miller. Another paper (No. 64) is entitled "Report on Groups of an Infinite Order." In this paper considerable attention is given to a memoir on groups of movements written by Jordan in 1868 and brief accounts are given of the work done by Lie, Klein, Dyck and others; this paper supplements the reports on groups of a finite order.

To present the nature of the work contained in the 104 papers reproduced in this volume and not mentioned above, it will be convenient to consider them under the headings: (1) Papers on the determination of groups of finite order, (2) Papers which deal with the theory of groups, (3) Papers on group theory and number theory, (4), Reviews; elementary and expository papers; applications. These will be considered in order.

\section{(1) Papers on the Determination of Groups of Finite Order.}

These papers will be considered under the subheadings (a) Groups of given degree or order, (b) Groups containing given subgroups, (c) Groups having given defining relations, (d) Groups having given groups of isomorphisms.

(a) Groups of given degree or order. The papers in Volume I on the determination of groups of finite order are concerned chiefly with permutation groups of given degree and abstract groups of given order. In Volume II there are only five such papers. In one of these (written with G. H. I.ing) a complete determination is made of the intransitive substitution groups of degree eleven. In another, all the abstract 
groups of order 168 are found. The other three are devoted to simple groups; in one of these (written with G. H. Ling) it is proved that there is no simple group whose order lies between 1902 and 2001, in another it is shown that the degree of a simple group of odd composite order must exceed fifty, and in a third the groups of isomorphisms of the simple groups whose degrees are less than fifteen are determined.

(b) Groups containing given subgroups. In the study of permutation groups, especially of those which are primitive, the subgroups which leave one element fixed have played a noteworthy part. In this volume several important papers are devoted to the study of abstract groups with given subgroups or given operators. Five of these deal with the determination of all groups of order $p^{m}$ ( $p$ a prime) which contain the Abelian group of type $(m-2,1)$, the Abelian group of order $p^{m-1}$ and type $(1,1, \cdots, 1)$, operators of order $p^{m-2}$, exactly $p$ cyclic subgroups of order $p^{\alpha}$, or exactly $p+1$ Abelian subgroups of order $p^{m-1}$. Two papers deal with groups of order $2^{m}$ which contain an invariant cyclic subgroup of order $2^{m-2}$, or an odd number of cyclic subgroups of composite order. An important set of papers deals with groups in which every subgroup is Abelian (this with H. C. Moreno), every subgroup of composite order is invariant, every subgroup is Abelian or Hamiltonian, every subgroup is Abelian or dihedral, or which contain less than six cyclic subgroups of the same order. Three other important papers are devoted to a generalization of the Hamiltonian groups, the invariant subgroups of prime index, and groups containing only three operators which are squares. Three papers relate to groups containing the largest possible number of operators of order two thirteen operators of order two, or less than fifteen operators of order two; one paper to the groups which contain less than twenty operators of order three; and one paper to the possible number of operators of order two in a group of order $2^{m}$. The remaining papers under this heading relate to the construction of a group of order $p^{\mathbb{e}}$ which includes no Abelian subgroup of order $p^{4}$, and to showing that each member of the Mathieu system of triply transitive groups is uniquely determined by its subgroup which leaves one element fixed.

(c) Groups having given defining relations. Much attention has been given by investigators in the field of group theory to the study of the properties of groups whose defining equations are given and the present volume contains a number of papers on this topic. One of the most important of these deals with the generalization of groups of genus zero. The generalization is obtained by replacing two of the three ordinary relations which define such a group by a single relation while the third is kept unchanged. Another paper closely allied to groups of genus zero is one on the groups which are generated by two operations of orders two and four respectively whose commutator is of order two.

Another paper of fundamental importance deals with groups defined by the orders $l, m$ of two generators and the order $n$ of their product. In this paper it is shown that there is always an infinite number of corresponding groups except when two of these numbers are equal to two, or one is two, the other three, while the third is three, four, or five. Papers relating to special cases deal with groups generated by two operators of order three whose product is also of order three, with groups generated by two operators of orders two and three respectively whose product is of order six, with groups generated by two operators of order three whose product is of order four, and with groups generated by two operators. Another paper deals with two infinite systems of groups generated by two operators of order four.

Another set of papers in this category is devoted to the groups generated by two operators which have a common square, groups generated by operators which trans- 
form each other into their powers, groups generated by two operators which transform each other into the same power, and groups generated by two operators such that each is transformed into its inverse by the square of the other. Another paper is devoted to groups generated by three operators each of which is the product of the other two.

(d) Groups having given groups of isomorphisms. In a paper in the Transactions devoted to this topic all possible groups are determined whose group of isomorphisms is either the symmetric group of order six, or the symmetric group of order twentyfour. The infinite system of groups is also determined whose group of cogredient isomorphisms is the symmetric group of order six.

\section{(2) Papers Which Deal with the Theory of Groups.}

These papers may be classified into those relating to permutation groups and those relating to abstract groups. Among the former that on the product of two substitutions is of fundamental importance by reason of the theorem: If $l, m, n$, are any three integers greater than unity, of which we call the greatest $k$, it is always possible to find three substitutions $(L, M, N)$ of $k+2$ or some smaller number of elements and of orders $l, m, n$ respectively such that $L M=N$. This theorem is the basis of several results on the determination of groups. In a second paper there is proved a fundamental theorem relating to the degrees of the transitive constituents of the Sylow subgroups of any transitive group. In another interesting paper it is shown that the group of the five times transitive function of 24 elements of Mathieu and its maximal subgroups of degrees 21,22 , and 23 are simple groups. Other papers on permutation groups relate to the transitive groups which are isomorphic to a given group, transitive groups of order $p^{m}$, primitive groups of class four, and the invariant substitutions under a substitution group.

The papers relating to the theory of abstract groups cover a wide range of topics. In one, on the groups which are the direct product of two subgroups, four important theorems are proved. In two others several new properties of the holomorph of a cyclic group are presented. Four papers relate to groups of isomorphisms; one to the group of isomorphisms of a cyclic group, one to that of a group of order $p^{m}$, and two to groups of isomorphism of Abelian groups. Three papers are on conformal groups; in one of these necessary and sufficient conditions are developed that any Abelian group be conformal with at least one non-Abelian group, in one an infinite system of non-Abelian groups which are not conformal with any Abelian group is studied, and in a third groups of order $p^{m}$ which are not conformal with Abelian groups are considered.

Several papers on abstract group theory relate to subgroups. Important among these is one in which all the characteristic subgroups of any Abelian group are determined, and another in which a formula is developed for writing all the subgroups of such a group. In one of two papers concerning groups of order $p^{m}$ it is proved that every non-Abelian group of this order contains at least $p$ invariant commutator operators, and its commutator quotient group is always non-cyclic (this being an extension of a theorem by Sylow); in the other it is shown that every non-cyclic group of order $p^{m}$ contains at least one invariant non-cyclic subgroup of order $p^{\beta}$, where $\beta>1$. In one of two papers concerned with the number of operators whose orders exceed two a formula is developed for this number in a non-Abelian group of order $2^{m}$ in which the number of operators of order 2 is more than half the order of the groups; in the other the minimum number of operators whose orders exceed two in any group of finite order is considered. Two papers relate to subgroups of order $p$ 
in groups of any given order; in one of them it is proved that if the order of a Sylow subgroup is $p^{m}$ and if this subgroup is non-cyclic then the number of cyclic subgroups of order $p^{\alpha}$ is $k p$, and in the other an upper limit is found for the number of sets of conjugate subgroups of order $p^{\alpha}$, where $\alpha<m$. Other papers concerning subgroups or operators are devoted to the invariant subgroups of index $p^{2}$, the number of Abelian subgroups whose order is a power of a prime, an addition to a theorem due to Frobenius, groups containing several operators of order two, and theorems relating to quotient groups.

Further papers on the theory of abstract groups are devoted to a method for constructing all groups of order $p^{m}$, to the roots of the operators of a group, to groups in which all the operators are contained in a series of subgroups such that any two have only the identity in common, to the commutators of a group of order $p^{m}$ and to the definition of a complete group. Besides these a number of theorems of importance are proved in the papers noted above on the determination of groups of finite order.

\section{(3) Papers on Group Theory and Number Theory.}

Six papers in this volume are devoted to the study of properties relating to both group theory and number theory. In a paper on holomorphisms and primitive roots important properties of the group of isomorphisms of a cyclic group of order $p^{m}$ are developed and used to prove the existence of primitive roots of $p^{m}$ and $2 p^{m}$ and also to show how to determine these roots. In another paper, theorems relating to the product of all the operators in an Abelian group and to the order of a cyclic group whose group of isomorphisms contains only one operator of order two provide the basis for a new proof of the generalized Wilson's Theorem. A third paper on some relations between number theory and group theory has close contact with the first paper above and others in the literature. Its chief object is to present certain additional developments which show the usefulness of group theory in the study of number theory. In two other papers elementary theorems in group theory are used to prove the formula for the totient of order $k$ with respect to $m$, and other well known relations connecting totients. The remaining paper in this category aims to translate several theorems in number theory into the language of group theory rather than to exhibit the advantages of employing theorems in group theory in the proof of important results in number theory as is done largely in the other five papers.

\section{(4) Reviews; Expository and Elementary Papers; Applications.}

Two papers are devoted to reviews of treatises on group theory. In one of these a rather complete review of Burnside's Theory of Groups (first edition) is given; in the other a brief review of Dickson's Linear Groups with an Exposition of the Galois Field Theory is made.

Expository and elementary papers relate to some methods of constructing substitution groups, the product of two commutative operators, the definition of an infinite number, the generalization and extension of Sylow's theorem, what is group theory, the commutator of two operators, a note on Netto's Theory of Substitutions, groups of the fundamental operations of arithmetic, and groups of subtraction and division.

Applications are given in papers on a new chapter in trigonometry, and the use of group theory in elementary trigonometry.

The last paper (No. 171) is entitled "Primary Facts in the History of Mathematics." It is a new paper written for this volume and, as stated at the beginning of 
this review, is included at Professor Miller's request. It presents some of the facts which guided him in his writings on the history of mathematics and affords a suitable basis for evaluating this phase of his work. The facts presented are discussed under the headings: abstractions, generalizations, wider outlook, special language, Greek contributions, thought currency, subjects treated, different treatments, fashions, independent discussions, formulas, and dependent subjects. This important paper will be of value and interest to students of the history of mathematics.

H. W. KuHN 